\title{
A system for the synthesis of nanoparticles by laser ablation in liquid that is remotely controlled with PC or smartphone
}

Cite as: Rev. Sci. Instrum. 90, 033902 (2019); https://doi.org/10.1063/1.5083811

Submitted: 30 November 2018 . Accepted: 26 February 2019 . Published Online: 18 March 2019

Simone Crivellaro, Andrea Guadagnini, David Muñetón Arboleda, Daniel Schinca, and Vincenzo Amendola (D)

CrossMark

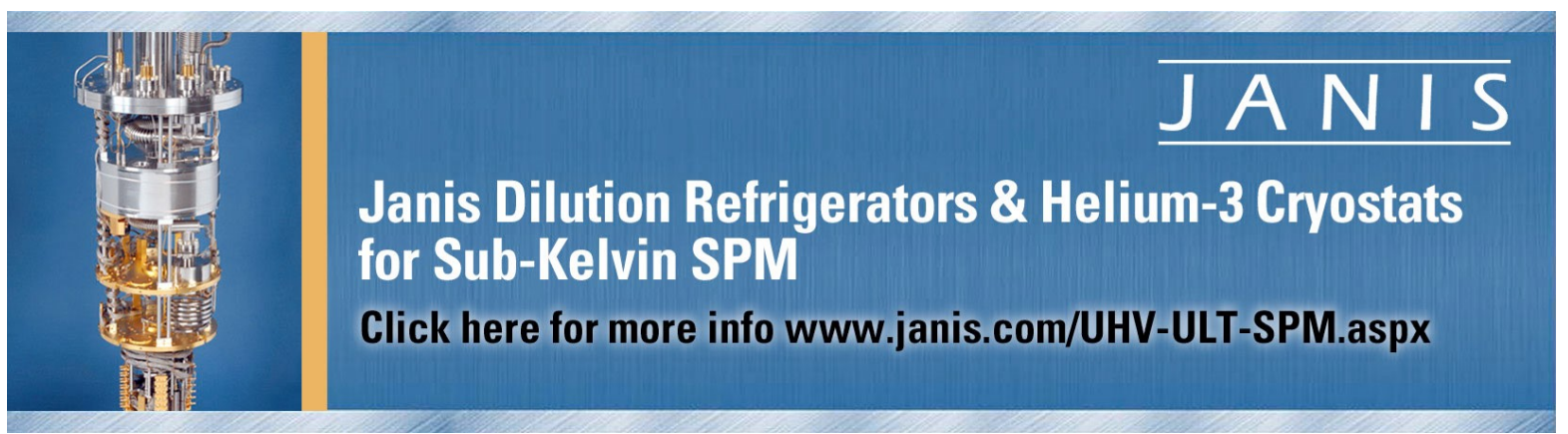




\title{
A system for the synthesis of nanoparticles by laser ablation in liquid that is remotely controlled with PC or smartphone
}

\author{
Cite as: Rev. Sci. Instrum. 90, 033902 (2019); doi: 10.1063/1.508381 1 \\ Submitted: 30 November 2018 - Accepted: 26 February 2019 • \\ Published Online: 18 March 2019
}

\author{
Simone Crivellaro, ${ }^{1}$ Andrea Guadagnini, ${ }^{1}$ David Muñetón Arboleda, ${ }^{2}$ Daniel Schinca, ${ }^{2}$ \\ and Vincenzo Amendola ${ }^{1, \text { a) }}$ (i)
}

\author{
AFFILIATIONS \\ ${ }^{1}$ Department of Chemical Sciences, University of Padova, Padova, Italy \\ ${ }^{2}$ Centro de Investigaciones Ópticas CIOp (CONICET-CIC-UNLP) and Facultad de Ingeniería UNLP, La Plata, Argentina
}

a)vincenzo.amendola@unipd.it

\begin{abstract}
Nanoparticles find applications in multiple technological and scientific fields, and laser ablation in liquid (LAL) emerged as a versatile method for providing colloidal solutions of nanomaterials with various composition, by a low cost, simple, self-standing, and "green" procedure. However, the use of high energy and high power laser beams is harmful, especially when coupled with flammable or toxic liquids, and in situ operation is required for starting, monitoring the LAL synthesis, and stopping it at the desired point. Here we describe the hardware and software design and the test results of a system for the production of nanoparticles by laser ablation synthesis in liquid solution (LASiS), which is remotely controllable with a personal computer or a smartphone. In this system, laser energy and solution flux are selectable, and the synthesis status can be monitored and managed at any time off site. Only commercially available components and software are employed, making the whole apparatus easily reproducible in any LAL laboratory. The system has proven its reliability in various conditions, including intercontinental remote control experiments. Overall, this apparatus represents a step forward to improve the safety and to more efficiently exploit the time of people working with LASiS, thus contributing to the increasing demand for off-site real time monitoring of experimental equipment in many scientific and industrial laboratories, due to safety and efficiency requirements.
\end{abstract}

Published under license by AIP Publishing. https://doi.org/10.1063/1.5083811

\section{INTRODUCTION}

Nanoparticles emerged as an indispensable tool for multiple technological products and scientific investigations, ranging from catalysis $^{7-3}$ to sensing, ${ }^{4}$ medicine,${ }^{5}$ and photonics. ${ }^{6,7}$ This is continuously prompting to the development of synthetic approaches which are of easy and general applicability and that allows the access to the largest possible class of nanomaterials, not just with the same procedure but even with the same experimental platform.

In this context, laser ablation synthesis in solution emerged as a versatile method for the production of nanoparticles dispersed in a liquid environment since it represents a close approximation to the widespread concept of coffee pods, where different flavors are obtained just by changing the capsule in the same machine. ${ }^{8,9}$ In fact, the basis of laser ablation synthesis in liquid solution (LASiS) consists in the production of nanoparticles as a colloidal solution by ablating with a pulsed laser beam a bulk target dipped in a liquid so that different types of nanomaterials are obtained just by changing the bulk target and/or the type of solution. ${ }^{10}$ LASiS with pulse durations from fs up to $\mathrm{ms}$ and even $\mathrm{cw}$ was reported in the literature although best productivities to date were observed for ps and ns pulses. $^{8,10}$

In laser ablation in liquid (LAL), the absorption of the pulsed laser beam by the target generates two shockwaves propagating in the underlying solid and the overlying liquid, while inducing the heating and ionization of the irradiated volume, which expands as a plasma plume toward the liquid solution on a time scale of picoseconds. ${ }^{11}$ The plasma plume expansion, which lasts for tens up to hundreds of ns, results in the heat transfer and vaporization of the liquid at its boundary, which proceeds up to the formation of a cavitation 
bubble after several hundreds of ns. ${ }^{11}$ On a time scale of $\mu \mathrm{s}$, the cavitation bubble expands and collapses, at least once, after which the matter extracted from the target is released into the liquid as a colloid. 8,10,11 In particular, according to latest computational studies, ${ }^{12}$ final nanoparticles are obtained from the coalescence of liquid and solid clusters ejected from the target by two mechanisms, which are the ejection of droplets due to an explosive boiling process, or the ejection of atoms due to target vaporization, which then nucleate in larger clusters due to supersaturation. Since the process takes place in the presence of vaporised or excited solution species, nanoparticles with composition different from that of the pristine target are also achievable, if the target is composed of reactive elements. 8,10 Importantly, nanoparticles' formation occurs on a time scale shorter than a $\mu$ s and all inside the cavitation bubble, which is especially well suited for the achievement of metastable phases requiring out of equilibrium synthetic conditions and fast temperature quenching to stabilize their structure and composition against thermodynamic driving forces. ${ }^{8,13}$ In fact, LASiS is becoming increasingly important for the achievement of metastable or unconventional nanomaterials such as alloys of immiscible elements. ${ }^{14-16}$

Additional appealing features of LASiS are the easy procedure, the low cost, the potentially "green" synthetic approach, and the access to nanoparticles with superior purity. 8,10,17-19 A remarkable key point is the limited manual operation since LASiS is a selfstanding synthesis where the operator is required only at the beginning and at the end of the process, except for routine control of its status.

Taking advantage from this feature, here we present a LASiS system developed with the ability of remote monitoring and controlling the main laser parameters through internet, just with a personal computer (PC) or a smartphone, and using the freeware software TeamViewer and the well known LabVIEW ${ }^{\mathrm{TM}}$ (Laboratory Virtual Instrument Engineering Workbench) environment.

Nowadays, internet-based monitoring and control entered in the common practice; however, in the case of LASiS, there are also other important reasons for off-site intervention, namely, avoiding human operation under harmful environmental circumstances. Because laser ablation in liquids sometimes can result in damaging of ablation cell and ignition of flammable solvents, in site human intervention may expose the operator to the danger of solvent explosion, free flames, and uncontrolled laser beam reflections.

Besides, the system is ideally suited for conducting the LASiS on highly toxic or radioactive species in a protected enclosure, without requiring local human intervention and avoiding the risk of contaminating the operator in the case of immediate intervention to interrupt the liquid flux and laser pulse generation. For instance, according to the International Atomic Energy Agency Safety Standards, the management of a wide class of materials with low or moderate radioactivity is possible by limiting the operator exposure time. ${ }^{20}$ The possibility of using a remotely controllable laser ablation setup for the production of radioactive nanomaterials is thus useful for limiting operator permanence close to the radioactive compounds. Besides, the European Agency for Safety and Health at Work alerts about exposure of operators in laboratories and factories to inhalation and/or contact with nanomaterials that may cause damage to workers present in the synthesis place. ${ }^{21}$

The remote control architecture is of general applicability and can be easily extended to other configurations such as LAL coupled with optical scanners for high throughput LASiS, laser fragmentation in liquid (LFL), or laser melting in liquid (LML).

\section{DESCRIPTION OF THE SYSTEM}

The remotely controlled LASiS system consists of a hardware and a software part.

The structure diagram for the hardware system is shown in Fig. 1. A Q-switched Nd:YAG laser (a) model Brilliant 50 from Quantel was used as the source for $1064 \mathrm{~nm}$ (6 ns) pulses with a repetition rate of $50 \mathrm{~Hz}$. Laser pulses were deviated with a mirror [(b), Thorlabs] and focused with an $\mathrm{f} 100 \mathrm{~mm}$ lens [(c), Thorlabs] into the



FIG. 1. Schematic diagram for the remotely controlled LASiS system: laser (a), mirror (b), lens (c), ablation cell (d), $X Y$ scanning stage (e), motor controller $(\mathrm{f})$, peristaltic pump (g), power supply (h), liquid reservoir (i), collection container (j), webcam/interlock (k), webcam (I), laptop PC (m), multiport USB interface ( $n)$, and RS232 to USB converter (o). Dashed box encloses components at the remote location: PC (p) or smartphone (q). 
custom-made ablation cell (d). The cell is mounted on a motorized $\mathrm{XY}$ scanning stage [(e), Standa] managed with a 2-axis stepper and DC motor controller [(f), Standa].

The cell is composed of two glass windows assembled on a Teflon spacer shaped to house the bulk target, which in our case is a disk (6 $\mathrm{mm}$ in diameter) or a plate ( $10 \mathrm{~mm}$ side). The Teflon spacer also supports inlet and outlet stainless steel tubes $(1 \mathrm{~mm}$ internal diameter). The inlet tube is connected with a silicon or a Tygon tube to a peristaltic pump [(g), Verderflex] with a flow rate selectable in the $1-8 \mathrm{ml} / \mathrm{min}$ or $6-60 \mathrm{ml} / \mathrm{min}$ range from a benchtop power supply [(h), RND 320-KD3005P], which sucks from a liquid reservoir (i). The outlet tube brings the laser generated colloid to a glass collection container (j). In this configuration (configuration 1), the liquid level in the reservoir or in the collection bottle can be monitored with a webcam $(\mathrm{k})$ coupled to an appropriate red-green-blue (RGB) analysis software, as described below. A webcam [(l), Trust] is used for real time video monitoring of the whole apparatus.

All the hardware components and controllers are connected to a laptop PC (m) through serial universal serial bus (USB) ports docking on a multiport USB interface (n) or, in the case of the laser source, through an RS232 to USB converter (o).

The hardware part is managed by the software platform sketched in Fig. 2, which runs under a Microsoft Windows OS (Windows 10 Professional 64 bit) and is designed to avoid conflicts in port identification and operative system crash or software slow downs. Because the laser system in use has an RS-232 serial port, we controlled the laser output using LabVIEW software according to RS232 protocol. LabVIEW (Laboratory Virtual Instrument Engineering Workbench) is the preferred platform for interfacing electronic instruments to a PC by using a serial connector, thus transforming them in "virtual instruments." 22 The DC power supply [(h) in Fig. 1] of the peristaltic pumps and the interlock webcam [(k) in Fig. 1] are also connected with a serial interface and are managed with the same LabVIEW program controlling the laser, while the DC motor controller [(f) in Fig. 1] is managed with an independent custom made LabVIEW program.

To avoid conflicts in port identification and operative system crash or software slows down, the laser, the pump, and the webcam are managed by a single LabVIEW routine, while the XY-stage is managed by a separate LabVIEW routine. Even in case of software slow down or crash and signal interruption, the XY-stage autonomously stops in its current position.

Therefore, as shown in Fig. 2, the platform consists of three independently running programs for the webcam (a), the triad composed by interlock (b), pump (c) and laser (d), and the XY scanning stage (e), plus TeamViewer (f).

The LabVIEW routine dedicated to the analysis of the webcam RGB signal can be used also for real time plotting of the instrument readout. When a preset threshold value is reached in the area selected by the operator, the program turns off the laser output and the DC voltage applied to the pump. Alternatively, a preset time can be provided as the interlock checkpoint to autonomously end the LASiS. Then, the same program sends an e-mail alert [(g) in Fig. 2] to the user account informing that the LASiS is completed or stopped.

The interlock webcam can be installed on the reservoir or on the collection bottle so that a sudden increase or decrease in the signal will correspond to the end of the solution. The interlock prevents laser ablation of the target in air in case the solution is finished, or the spill over of liquid when mounted on the collection bottle. The interlock threshold is sensitive to the illumination conditions in the laboratory, which can be fixed by using a normal lamp as the artificial light source, and the area used for the interlock is selected around the reservoir bottles. The criteria for selection of this area rely on the homogeneity of the colour on the surface of the bottle and the absence of reflections due to the illumination sources such as lamps or windows.

In a different configuration (configuration 2), where just one bottle is used for LASiS and the same solution is fluxed multiple times through the cell until reaching the desired NP concentration, the interlock can be used to run the LASiS until reaching the desired concentration of NPs. In fact, the nanoparticle concentration in the liquid is related to its optical extinction, which corresponds to an increasing value in the appropriate RGB channel (for instance, the red channel for Au colloids)

Finally, the webcam video is accessed with the built-in utility available in the Windows OS.

The software platform can be managed by the PC in site or off site by using any PC or smartphone [(p) and (q) in Fig. 1] with the software TeamViewer [(h) in Fig. 2] installed.

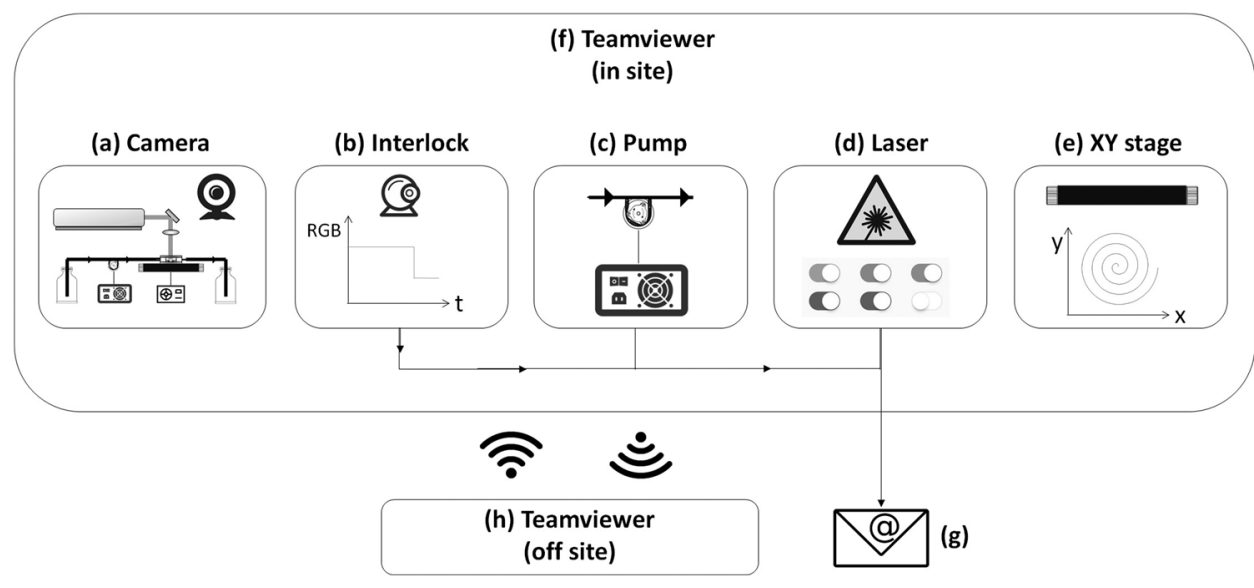

FIG. 2. Schematic diagram of the software platform for the control of the hardware LASiS apparatus: webcam monitoring (a); control of laser, pump, and interlock [(b)-(d)]; and XY scanning stage (e). Everything can be managed remotely by the TeamViewer software active in site ( $f$ ) and in the off site device (h). An e-mail (g) is sent when laser and pump are stopped by the interlock. 
All the hardware apparatus, including the laser source, was mounted on an optical breadboard measuring $40 \mathrm{~cm}$ per $110 \mathrm{~cm}$ of length. Therefore, it can be easily positioned on an independent, optically screened, movable cart, for executing the synthesis in the required location, such as a room dedicated to the manipulation of toxic or radioactive compounds, where human presence must be limited and subject to strict security procedures.

\section{TEST RESULTS}

The remote controlled LASiS system has been tested using two synthesis environments, an aqueous solution of $\mathrm{NaCl}\left(10^{-4} \mathrm{M}\right.$ in distilled water) or an ethanol solution of polyvinylpyrrolidone $0.1 \mathrm{mg} / \mathrm{ml}$ [PVP, Sigma-Aldrich, in high-performance liquid chromatography (HPLC) grade ethanol from Sigma Aldrich]. In these experiments, the metal targets were composed of single elements such as $99.9 \%$ pure $\mathrm{Au}$ and $\mathrm{Ag}$, or alloy targets of $\mathrm{Au}-\mathrm{Ag} 1: 1$ and $\mathrm{Au}-\mathrm{Fe}$ 75:25.

Figure 3 shows the typical screenshots as they appear on the remote $\mathrm{PC}$ or on a smartphone when TeamViewer is adopted for the connection. The main controls related to the pump and the laser, and the camera interlock, are preferentially kept in foreground, while the panoramic camera view and the XY translations stage routine are in background and can be recalled when necessary.

The LASiS system was remotely operated without failure from the same building, from international locations such as during international conference, ${ }^{23}$ and from transatlantic laboratories, such as from CIOp (Argentina) by controlling the setup located at the University of Padova. The response time to the command of laser shut off with the LabVIEW program is of $10^{-3} \mathrm{~s}$, measured with LabVIEW "Timing" function. Concerning the pace of information update with TeamViewer, in our experimental conditions, the program required a bandwidth of the order of $0.1-10 \mathrm{kbytes} / \mathrm{s}$, depending on screen resolution and desired connection speed; therefore, communications on a time scale of fraction of seconds will be possible with an Internet connection with a bandwidth of 1-0.1 Mbytes/s or larger.

As a demonstration of system reliability, Fig. 4(a) shows the UV-visible absorption spectra of the resulting output solutions synthesized remotely in different operating conditions such as with $\mathrm{Au}$ (red line) and Ag (dark yellow line) targets in an aqueous environment with a fluence of $5 \mathrm{~J} / \mathrm{cm}^{2}$. The two spectra clearly show the surface plasmon absorption peak typical of spherical colloids of $\mathrm{Au}$ and Ag dispersed in water, respectively, peaked at $520 \mathrm{~nm}$ and $400 \mathrm{~nm}$, as previously reported in several studies. ${ }^{24-27}$ The TEM analysis [Figs. 4(c) and 4(d)] shows that nanoparticles have the expected spherical shape with an average size of $11 \pm 6 \mathrm{~nm}$ (Au NPs) and $20 \pm 8 \mathrm{~nm}$ (Ag NPs). ${ }^{28}$ The Au colloids was achieved in configuration 1 , by setting the interlock on the "white" RGB channel and selecting a $4 \mathrm{~cm} \times 4 \mathrm{~cm}$ area on the bottom of the reservoir bottle [(i) in Fig. 1], containing the aqueous solution and a white Teflon float. When the liquid level approached the bottom of the bottle, the Teflon float entered in the area selected for the interlock, which autonomously stopped the LASiS. The Ag colloid

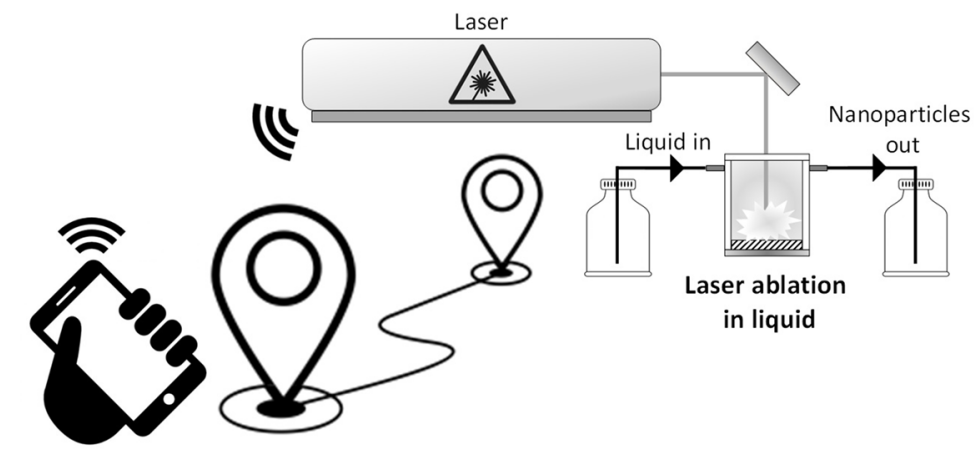

Smartphone screenshot



PC screenshot

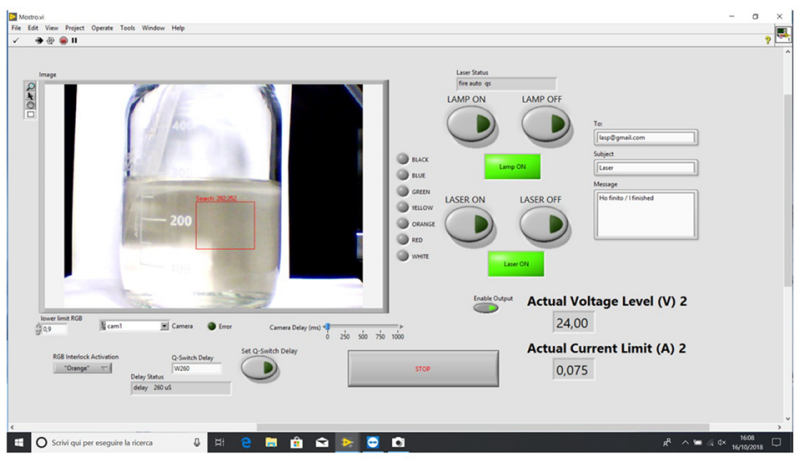

FIG. 3. Typical screenshot of the operating panels selected for remote monitoring and control of LASiS with a smartphone (left) or a PC (right). 



B

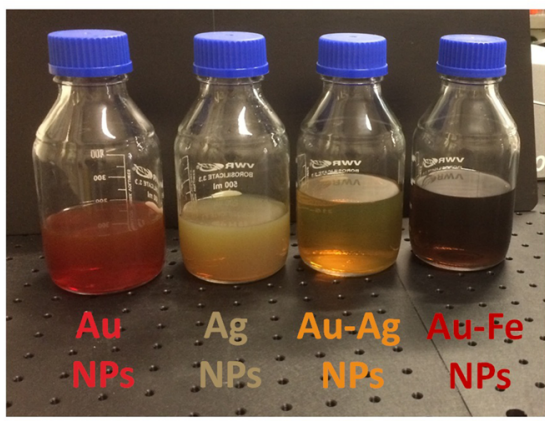

FIG. 4. (a) UV-Vis spectra of $\mathrm{Au}$ (red line) and Ag (yellow line) colloids in aqueous solution and of Au-Ag (orange line) and Au-Fe (brownish line) colloid in PVP solution in ethanol, all obtained by off-site LASiS. (b) The bottles containing the colloidal solutions collected at the end of the synthesis: from left to right: $\mathrm{Au}, \mathrm{Ag}, \mathrm{Au}-\mathrm{Ag}$, and $\mathrm{Au}-\mathrm{Fe}$. [(c)-(f)] Representative TEM images for $\mathrm{Au}(\mathrm{c}), \mathrm{Ag}(\mathrm{d}), \mathrm{Au}-\mathrm{Ag}(\mathrm{e})$, and $\mathrm{Au}-\mathrm{Fe}(\mathrm{f}) \mathrm{NPs}$.

was obtained in configuration 2 , by setting the interlock on the "yellow" RGB channel and selecting the probed area in the middle of the unique bottle used in this configuration. Therefore, the LASiS ended autonomously as soon as the solution turned yellow, according to the RGB analysis algorithm. Higher NP concentration can be achieved by using the alternative interlock checkpoint consisting in a preset LASiS duration (such as $60^{\prime}$ ).

Figure 4(a) also shows the absorption spectra in the case of Au-Ag 1:1 (orange line) and Au-Fe 7:3 (brownish line) alloy targets ablated in the ethanol solution of PVP with the same fluence of $5 \mathrm{~J} / \mathrm{cm}^{2}$. Also in these cases, the optical properties are distinctive of the composition. In fact, the Au-Ag colloid shows an absorption peak at $464 \mathrm{~nm}$, which is exactly the intermediate wavelength between that of the two pure metals. This is in agreement with the value expected for Au:Ag 1:1 nanoalloys, according to the general observation that the plasmon band energy shifts in a nearly proportional way with the amount of the two metals in the nanoalloys. ${ }^{29}$ In the case of the Au-Fe nanoalloys, it was demonstrated that the surface plasmon band is damped compared to that of pure Au NPs, ${ }^{30}$ while Fe NPs do not support a plasmon in the visible range, and in fact the UV-Vis spectrum of the PVP-coated Au-Fe nanoalloys in Fig. 4(a) is almost flat. This corresponds to a brownish colour of the solution as it is appreciable by the naked eye in Fig. 4(b) reporting the picture of the collection bottles at the end of the four syntheses. TEM analysis evidenced the regular spherical shape of the $\mathrm{Au}-\mathrm{Fe}$ NPs, with an average size of $7 \pm 6 \mathrm{~nm}$.

Given the optical properties of the $\mathrm{Au}-\mathrm{Ag}$ and $\mathrm{Au}-\mathrm{Fe}$ nanoparticles, the interlock was set to the orange and black RGB channels, respectively, and the synthesis ended autonomously when this colour was detected from the camera.

\section{CONCLUSIONS}

Nowadays, the demand for off-site real time monitoring of experimental equipment is increasing in many scientific and industrial laboratories, due to safety and efficiency requirements. Here we showed the application of remote control to the synthesis of nanoparticles by the LASiS technique, with an apparatus consisting of a hardware and a software part. The hardware system can be controlled off site with a PC or a smartphone and exploits commercially available components. The software platform is user-friendly, interactive, and only requires a cost-effective computer and freeware or widespread commercial software such as TeamViewer and LabVIEW. The system was successfully tested for the off site synthesis of metal nanoparticles.

So far, nanomaterials obtained by LASiS have been applied in a long series of technological applications and basic science studies, not only for the ease of synthesis but also for their peculiar properties such as high purity and appealing multifunctional metastable phases. With the system architecture described here, the LASiS does not need the in site human operation in case of critical conditions requiring immediate laser shut down such as explosions, flames, and uncontrolled reflections of the laser beam, as well as in case of synthesis with harmful or radioactive compounds in rooms with restricted access for security reasons. 
In general, the remote control allows a more efficient management of LASiS operator time, without eliminating the possibility of effective control on the crucial system parameters. In principle, it makes LASiS accessible to any operator, while laser experts are required only for the laser alignment procedure.

We expect that this architecture will be applied also to other laser-assisted synthesis systems such as laser fragmentation in liquid (LFL), laser melting in liquid (LML), or high throughput LAL with the use of optical scanners.

\section{ACKNOWLEDGMENTS}

This research was performed with the support of the University of Padova STARS grant "4NANOMED" and "Iniziative di Cooperazione Universitaria 2017," and the Ministry of Foreign Affairs and International Cooperation (General Directorate for the Promotion of the Country System) "Great relevance project" with Protocol No. 0191594

\section{REFERENCES}

${ }^{1}$ B. M. Hunter, H. B. Gray, and A. M. Müller, "Earth-abundant heterogeneous water oxidation catalysts," Chem. Rev. 116, 14120-14136 (2016).

${ }^{2}$ S. Hu, M. Tian, E. L. Ribeiro, G. Duscher, and D. Mukherjee, “Tandem laser ablation synthesis in solution-galvanic replacement reaction (LASiS-GRR) for the production of PtCo nanoalloys as oxygen reduction electrocatalysts," J. Power Sources 306, 413-423 (2016).

${ }^{3}$ P. Liu, W. Cai, M. Fang, Z. Li, H. Zeng, J. Hu, X. Luo, and W. Jing, "Room temperature synthesized rutile $\mathrm{TiO}_{2}$ nanoparticles induced by laser ablation in liquid and their photocatalytic activity," Nanotechnol. 20, 285707 (2009).

${ }^{4}$ K. M. Mayer and J. H. Hafner, "Localized surface plasmon resonance sensors," Chem. Rev. 111, 3828-3857 (2011).

${ }^{5}$ J. Shi, P. W. Kantoff, R. Wooster, and O. C. Farokhzad, "Cancer nanomedicine: Progress, challenges and opportunities,” Nat. Rev. Cancer 17, 20-37 (2017).

${ }^{6}$ A. F. Koenderink, A. Alù, and A. Polman, "Nanophotonics: Shrinking light-based technology," Science 348, 516-521 (2015).

${ }^{7}$ C. Doñate-Buendia, R. Torres-Mendieta, A. Pyatenko, E. Falomir, M. FernándezAlonso, and G. Mínguez-Vega, "Fabrication by laser irradiation in a continuous flow jet of carbon quantum dots for fluorescence imaging," ACS Omega 3, 2735-2742 (2018).

${ }^{8} \mathrm{~V}$. Amendola and M. Meneghetti, "What controls the composition and the structure of nanomaterials generated by laser ablation in liquid solution?," Phys. Chem. Chem. Phys. 15, 3027-3046 (2013).

${ }^{9} \mathrm{~V}$. Amendola and S. Barcikowski, "A quarter-century of nanoparticle generation by lasers in liquids: Where are we now, and what's next?,” J. Colloid Interface Sci. 489, 1-2 (2017).

${ }^{10}$ D. Zhang, B. Gökce, and S. Barcikowski, "Laser synthesis and processing of colloids: Fundamentals and applications," Chem. Rev. 117, 3990-4103 (2017).

${ }^{11}$ J. Lam, J. Lombard, C. Dujardin, G. Ledoux, S. Merabia, and D. Amans, "Dynamical study of bubble expansion following laser ablation in liquids," Appl. Phys. Lett. 108, 074104 (2016).

${ }^{12}$ C.-Y. Shih, C. Wu, M. V Shugaev, and L. V. Zhigilei, "Atomistic modeling of nanoparticle generation in short pulse laser ablation of thin metal films in water," J. Colloid Interface Sci. 489, 3 (2017).
${ }^{13}$ D. Zhang, J. Liu, and C. Liang, "Perspective on how laser-ablated particles grow in liquids," Sci. China Phys., Mech. Astron. 60, 074201 (2017).

${ }^{14}$ V. Amendola, S. Scaramuzza, F. Carraro, and E. Cattaruzza, "Formation of alloy nanoparticles by laser ablation of $\mathrm{Au} / \mathrm{Fe}$ multilayer films in liquid environment," J. Colloid Interface Sci. 489, 18-27 (2017).

${ }^{15}$ S. Scaramuzza, S. Agnoli, and V. Amendola, "Metastable alloy nanoparticles, metal-oxide nanocrescents and nanoshells generated by laser ablation in liquid solution: Influence of the chemical environment on structure and composition," Phys. Chem. Chem. Phys. 17, 28076-28087 (2015)

${ }^{16}$ S. Scaramuzza, D. Badocco, P. Pastore, D. F. Coral, M. B. Fernández van Raap, and V. Amendola, "Magnetically assembled SERS substrates composed of ironsilver nanoparticles obtained by laser ablation in liquid," ChemPhysChem $\mathbf{1 8}$, 1026 (2017).

${ }^{17}$ B. Gökce, V. Amendola, and S. Barcikowski, "Opportunities and challenges for laser synthesis of colloids," ChemPhysChem 18, 983-985 (2017).

${ }^{18}$ J. Zhang, J. Claverie, M. Chaker, and D. Ma, "Colloidal metal nanoparticles prepared by laser ablation and their applications," ChemPhysChem 18, 986 (2017).

${ }^{19}$ V. Amendola, L. Litti, and M. Meneghetti, "LDI-MS assisted by chemical-free gold nanoparticles: Enhanced sensitivity and reduced background in the low-mass region,” Anal. Chem. 85, 11747-11754 (2013).

${ }^{20}$ IAEA PUBLICATIONS, Basic Safety Standards, GSR part 3 (2014), STI/PUB/ 1578 .

${ }^{21}$ S. Kaluza, J. Balderhaar, B. Orthen, E. Jankowska, P. Pietrowski, M. Rosell, C. Tanarro, J. Tejedor, and A. Zugasti, in Workplace Exposure to Nanoparticles [European Agency for Safety and Health at Work (EU-OSHA), 2009], https://osha.europa.eu/en/publications/literature_reviews/workplace_exposure_ to_nanoparticles/view.

${ }^{22}$ I. W. Kirkman and P. A. Buksh, "Data acquisition and control using national instruments' 'LabVIEW' software,” Rev. Sci. Instrum. 63, 869-872 (1992).

${ }^{23}$ V. Amendola, "Laser synthesis of 'pure' and 'doped' gold nanoparticles," in Gold 2018 Conference Paris, July 15-18, 2018.

${ }^{24}$ V. Amendola, R. Pilot, M. Frasconi, O. M. Maragò, and M. A. Iatì, "Surface plasmon resonance in gold nanoparticles: A review," J. Phys. Condens. Matter. 29, 203002 (2017).

${ }^{25}$ V. Amendola, O. M. M. Bakr, and F. Stellacci, "A study of the surface plasmon resonance of silver nanoparticles by the discrete dipole approximation method: Effect of shape, size, structure, and assembly," Plasmonics 5, 85-97 (2010).

${ }^{26}$ J. M. J. Santillán, M. B. F. van Raap, P. M. Zélis, D. Coral, D. Muraca, D. C. Schinca, and L. B. Scaffardi, "Ag nanoparticles formed by femtosecond pulse laser ablation in water: Self-assembled fractal structures," J. Nanoparticle Res. 17, 1-13 (2015).

${ }^{27}$ J. M. J. Santillán, L. B. Scaffardi, and D. C. Schinca, "Quantitative optical extinction-based parametric method for sizing a single core-shell $\mathrm{Ag}-\mathrm{Ag}_{2} \mathrm{O}$ nanoparticle," J. Phys. D.: Appl. Phys. 44, 105104 (2011).

${ }^{28} \mathrm{~V}$. Amendola and M. Meneghetti, "Laser ablation synthesis in solution and size manipulation of noble metal nanoparticles," Phys. Chem. Chem. Phys. 11, 3805-3821 (2009).

${ }^{29}$ S. Link, Z. L. Wang, and M. A. El-Sayed, "Alloy formation of gold-silver nanoparticles and the dependence of the plasmon absorption on their composition," J. Phys. Chem. B 103, 3529-3533 (1999).

${ }^{30}$ V. Amendola, S. Scaramuzza, S. Agnoli, S. Polizzi, and M. Meneghetti, "Strong dependence of surface plasmon resonance and surface enhanced Raman scattering on the composition of Au-Fe nanoalloys," Nanoscale 6, 1423-1433 (2014). 